Journal of Engineering Science and Technology Review 8 (2) (2015) 37-43

Special Issue on Synchronization and Control of Chaos: Theory, Methods and Applications

Research Article

\title{
Noise Separation from the Weak Signal Chaotic Detection System
}

\author{
Hanjie Gu* and Fan Wu \\ Institute of Information Technology, Zhejiang Shuren University, Hangzhou, 310014, Zhejiang, China.
}

Received 27 September 2014; Revised 14 October 2014; Accepted 18 November 2014

\begin{abstract}
The traditional weak signal chaotic detection system still restricts some technical issues in the situation of the signal with noise, such as poor denoising ability and low detection precision. In this paper, we propose a novel weak signal chaotic detection system based on an improved wavelet transform algorithm. First, the traditional wavelet transform algorithm domain variables have been transformed and discretized to eliminate the redundant transform. Then, based on the discrete optimization, the wavelet coefficients have been optimized by threshold compromise strategy. The improved wavelet transform algorithm is applied in the weak signal chaotic detection system. The noise signal after finite discrete processing is treated as a perturbation of cycle power and put into a chaotic system for detecting weak signal under the noise conditions. The simulation experiments show that the proposed improved wavelet transform algorithm has a better denoising effect than the traditional wavelet transform algorithm. Moreover, the improved algorithm shows better accuracy and higher robustness in the weak signal chaotic detection system.
\end{abstract}

Keywords: Weak Signal Detection, Chaotic System, Noisy Signals, Improved Wavelet Transform Algorithm, Discrete Optimization, Threshold Compromise Strategy.

\section{Introduction}

In the long-term production practice and scientific researches, people would like to know how to extract interested signals from noise background [1]. The problem is summed up as weak signal detection and estimation. The technology and methods to extract useful signal from noise is the main content of the study of weak signal detection [2]. It uses the information theory, electronics, physics and mathematical statistics method, etc. to analyze the characteristics and regular patterns of noise and valuable information. Then, it uses appropriate signal processing methods to extract the useful information from strong noise. It can also be used for detecting the the indicators of signal and the noise [3]. Therefore, weak signal detection technology plays an important role in the development of high and new technology, exploration and discovery of new natural law, promoting the development of science and technology and the production [4].

The commonly used conventional signal detection methods include power spectral analysis, wavelet transform, adaptive filter, the relevant test, synchronous accumulative method and signal to noise space decomposition. However, these methods are mainly based on linear system. The target signal extraction is often accomplished by amplification of useful signal through suppressing noise conditions [5]. Klein electronic technology method accomplished digital

* E-mail guhanjie82@yeah.net ISSN: 1791-2377 @ 2015 Kavala Institute of Technology. All rights reserved. average and integral sampling for the first time. However, the method has to know the signal frequency before the algorithm implementation. Thus, it will not be able to realize the signal cycle division if the signal frequency is unknown. It also will not be able to take average or digital sample of signals under the same temporal interval with different periods [6]. Weinred reported a detection method which implements the process of periodic signal extracted from random noise background. This method only requires a small amount of calculation with simple principles. It has been widely used in the engineering practice. The drawback of this method is that it loses signal phase information after signal processing [7]. Fourier proved that any periodic signal is a developable form into a series of sine functions, which makes Fourier transform method is widely used in the field of weak signal detection and estimation. However, this method has poor performance under the low signal noise ratio, which prone to make frequency spectrum leakage and fence effect, leading to the non-accurate detection [8]. Because chaotic system has a high sensitivity to the infinitesimal changes in the initial value and perturbation, so it is widely used in the weak signal detection [9]. Li [10] improved the Duffing oscillator and used the Melnikov function to analyze the principle and method of the phase transition of the chaotic system, which reaches to the detection of limit of $-111.46 \mathrm{~dB}$ under colored noise background. Lu et al. [11] reported a weak signal amplitude estimation method regards to the sensitive dependence for parameters of chaotic system. This method uses the least square method to find the relationship 
between the weak signal amplitude and Duffing under the condition of known signal frequency, and gives the mathematical expression of the amplitude calculation. Chen et al. [12] combined the cross-correlation algorithm with chaotic detection method, proposing a weak signal detection method based on the cross-correlation method and chaos theory. The experimental results show that with the combination of cross-correlation algorithm makes chaos detection obtain a better performance. $\mathrm{Li}$ et al. [13] proposed a weak signal detection method based on the wavelet transformation and chaos theory. This method puts the pretreated signals by wavelet transform into chaos detector and estimates the amplitude of the sine signal under the signal-to-noise ratio of $-40 \mathrm{~dB}$. This method has been used for collecting the electric shock signal detection. Wang et al. [14] analyzed the basic principle and feasibility of chaotic oscillator detection of a weak signal under strong noise background and dentally studied the features of noise. The results indicate the advantages of using chaos algorithm to determine the signal. Chance and Scott [15] used the perturbation of chaotic trajectory control the weak signal detection of the microwave system. Although, the above methods can detect the weak signal, but under the strong noise or the condition of destroyed chaotic phase space structure, these methods remain the defects of accuracy.

This article in view of the existing problems of weak signal chaotic detection system after adding noise, proposes a chaotic detection system based on improved wavelet transforms. This method uses discrete optimization and compromise strategies for optimizing threshold wavelet transform algorithm and improves the denoising ability of weak signal detection chaotic system.

\section{Weak Signal Chaotic Detection System under Noise Condition Proposed Circuit}

The Duffing oscillator chaotic system has been widely used in weak signal detection due to its simple equation structure and low order. Duffing equation can be shown as:

$$
\ddot{x}(t)+k \dot{x}(t)-x(t)+x^{3}(t)=a \cos \omega t
$$

where $k$ is damping ratio, $\alpha$ is cycle power amplitude, $-x+x^{3}$ is nonlinear elastic force, $\omega$ is cycle policy dynamics. The formula (1) can be converted to the firstorder differential equations.

$$
\left\{\begin{array}{l}
\dot{x}=y \\
\dot{y}=x-x^{3}+a \cos \omega t-k y
\end{array}\right.
$$

In order to measure any frequency periodic signal, the Duffing equation has to be improved because the frequency of the periodic signal under weak signal detection is changing. For equation of Duffing chaotic systems:

$\ddot{x}+k \dot{x}-x+x^{3}=\gamma \cos \omega t$

Let $t=\omega \lambda$, then $x(t)=x(\omega \lambda)$. Then:

$$
x(t)=x(\omega \lambda)
$$

and

$$
\begin{gathered}
\dot{x}(t)=\frac{d x(t)}{d t} \\
=\frac{d x(\omega \lambda)}{d(\omega \lambda)} \\
=\frac{1}{\omega} \dot{x}(\omega \lambda)
\end{gathered}
$$

or

$$
\begin{aligned}
& \dot{x}(t)=d\left[\frac{1}{\omega} \cdot \dot{x}(\omega \lambda)\right] / d(\omega \lambda) \\
& =\frac{1}{\omega^{2}} \ddot{x}(\omega \lambda)
\end{aligned}
$$

Put Eqs. (4) and (5) into Duffing equation, then:

$$
\frac{1}{\omega^{2}} \ddot{x}(\omega \lambda)+\frac{k}{\omega} \dot{x}(\omega \lambda)-x(\omega \lambda)+x^{3}(\omega \lambda)=\gamma \cos (\omega \lambda)
$$

Equation (7) is a system equation based on the independent variable $\lambda$. The equation of state can be changed as follows:

$$
\ddot{x}=-\omega k \dot{x}+\omega^{2}\left(x-x^{3}+\gamma \cos \omega \lambda\right)
$$

Results derived from the equation show that the threshold of Duffing chaotic system only relates with $\omega$. When the signal frequency changes, the only value should be changed is $\omega$.

The border cycle track of weak signal chaos detection system under noise condition is rough. Use $\Delta x(t)$ as the microvariations to $x(t)$, thus it is concluded that in the differential equation of the system under the noise condition. Eq. (3) can be expressed as:

$$
\begin{array}{r}
(\ddot{x}+\Delta \ddot{x})+k(\dot{x}+\Delta \dot{x})-(x+\Delta x)^{3}+(x+\Delta x)^{5}= \\
=\gamma \cos (\omega t)+n(t)
\end{array}
$$

where $n(t)$ is noise, $E\{n(t)\}=0$. Equation (9) minus Equation (3), because the value of $\Delta x$ is tiny, it can omit its high-order:

$\Delta \ddot{x}+k \Delta \dot{x}+3 x^{2} \Delta x-5 x^{4} \Delta x=n(t)$

Let $c(t)=5 x^{4}-3 x^{2}$ then,

$\Delta \ddot{x}+k \Delta x-c(t) \Delta x=n(t)$

Express Eq. (11) to vector differential equation:

$$
\dot{X}(t)=A(t) X(t)+N(t)
$$

and

$$
\left\{\begin{array}{l}
X(t)=\left[\begin{array}{l}
x_{1} \\
x_{2}
\end{array}\right]=\left[\begin{array}{l}
\Delta x(t) \\
\Delta \dot{x}(t)
\end{array}\right] \\
A(t)=\left[\begin{array}{ll}
0 & 1 \\
c(t) & -k
\end{array}\right] \\
N(t)=\left[\begin{array}{c}
0 \\
n(t)
\end{array}\right]
\end{array}\right.
$$


Its solution is $X(t)=\Phi\left(t, t_{0}\right) X_{0}+\int_{t_{0}}^{t} \Phi(t, u) N(u) d u$, where

$\Phi$ is the system state transition matrix. Due to the first solution is the transient solution, which will soon decay to zero, so only the second solution needs to be considered. Therefore:

$$
\left\{\begin{array}{l}
X(t)=\int_{t_{0}}^{t} \Phi(t, u) N(u) d u \\
E\{X(t)\}==\int_{t_{0}}^{t} \Phi(t, u) E\{N(u)\} d u=0
\end{array}\right.
$$

In the sense of statistics, any zero-mean noise will not change the original trajectory of the system, they will only make the system trajectory become rough and swing near the ideal trajectory.

\section{Optimization of Weak Signal Chaotic Detection System}

\subsection{Discrete Optimization of Wavelet Transform Algorithm}

Wavelet analysis is the expansion of Fourier analysis. Let $\forall f(t) \in L^{2}(R)$, the wavelet transform of $f(t)$ can be defined as:

$$
W T_{f}(a, b)=|a|^{-1 / 2} \int_{-\infty}^{\infty} f(t) \psi\left(\frac{t-b}{a}\right) d t, a \neq 0
$$

Or use the inner product form:

$$
W T_{f}(a, b)=\left\langle f, \psi_{a, b}\right\rangle
$$

In the equation:

$$
\psi_{a, b}(t)=|a|^{-1 / 2} \psi\left(\frac{t-b}{a}\right)
$$

To make the inverse transformation, $\psi(t)$ has to be satisfied:

$C_{\psi}=\int_{-\infty}^{\infty} \frac{|\hat{\psi}(\omega)|^{2}}{|\omega|} d \omega<\infty$

In the equation, $\hat{\psi}(\omega)$ is the Fourier transform of $\psi(t)$. The inverse transformation is:

$$
f(t)=C_{\psi}^{-1} \int_{-\infty}^{\infty} \int_{-\infty}^{\infty} \psi_{a, b}(t) W T_{f}(a, b) d b \frac{d a}{|a|^{2}}
$$

Constant $C_{\psi}$ restricts the species of $L^{2}(R)$ of $\psi$ function (as mother wavelet). If the $\psi$ is a window function, thus the $\psi$ should belong to $L^{1}(R)$. So:

$$
\int_{-\infty}^{\infty}|\psi(t)| d t<\infty
$$

$\hat{\psi}(\omega)$ is a continuous function of $R$. From equation (18), the $\hat{\psi}$ value is 0 at the origin. Then:

$\hat{\psi}(0)=\int_{-\infty}^{\infty} \psi(t) d t=0$

The equation (20) indicates the wavelet function has inevitable oscillation.

For the continuous wavelet transform is redundant, transform domain variables $\alpha$ and $b$ has to be discretized to remove the redundant in the transformation. In practice, the value of variables are usually chosen as $b=\frac{k}{2^{j}}, a=\frac{1}{2^{j}}$ and $j, k \in Z$. Then:

$\psi_{a, b}(t)=\psi_{\frac{1}{2^{j}}, \frac{k}{2^{j}}}(t)=2^{j / 2} \psi\left(2^{j} t-k\right)$

Often abbreviated to: $\psi_{j, k}(t)$.

The transformation can be expressed as: $W T_{f}\left(\frac{1}{2^{j}}, \frac{k}{2^{j}}\right)=\left\langle f, \psi_{j, k}\right\rangle$.

In order to reconstruct the signal $f(t),\left\{\psi_{j, k}\right\}_{j, k \in z}$ has to be a Riesz base of $L^{2}(R)$.

Function $\psi \in L^{2}(R)$ is a $R$ function, if $\left\{\psi_{j, k}\right\}_{j, k \in z}$ is a Riesz base in the following definition: if $\left\{\psi_{j, k}\right\}_{j, k \in z}$ has a dense linear $r$. vishny in $L^{2}(R)$ and there are normal numbers $A$ and $B(0<A \leq B<\infty)$. Then:

$$
A\left\|\left\{c_{j, k}\right\}\right\|_{l^{2}}^{2} \leq\left\|\sum_{j \in-\infty}^{\infty} \sum_{k \in-\infty}^{\infty} c_{j, k} \psi_{j, k}\right\|_{2}^{2} \leq B\left\|\left\{c_{j, k}\right\}\right\|_{l^{2}}^{2}
$$

For all the double infinite square and sequence $\left\{c_{j, k}\right\}$ can be established, then it fits to the $\left\|\left\{c_{j, k}\right\}\right\|_{l^{2}}^{2}=\sum_{j \in-\infty}^{\infty} \sum_{k \in-\infty}^{\infty}\left|c_{j, k}\right|^{2}<\infty$.

Suppose $\psi$ is a function $R$, then there is only one Riesz base $\left\{\psi^{j, k}\right\}_{j, k \in z}$ in $L^{2}(R)$.

$\left\langle\psi_{j, k}, \psi^{l, m}\right\rangle=\delta_{j, l} \delta_{k, m}, j, k, l, m \in Z$

If the equation is the dual of $\left\{\psi_{j, k}\right\}$, then every $f(t) \in L^{2}(R)$ has only one series according to the equation (21):

$$
f(t)=\sum_{j=-\infty}^{\infty} \sum_{k=-\infty}^{\infty}\left\langle f, \psi_{j, k}\right\rangle \psi^{j, k}(t)
$$

Especially, if $\left\{\psi_{j, k}\right\}_{j, k \in z}$ is $L^{2}(R)$ standard orthogonal basis, then $\psi_{j, k}=\psi^{j, k}$. The reconstruction formula can be expressed as: 


$$
f(t)=\sum_{j=-\infty}^{\infty} \sum_{k=-\infty}^{\infty}\left\langle f, \psi_{j, k}\right\rangle \psi_{j, k}(t)
$$

\subsection{Threshold Optimization of Discrete Wavelet Transform Algorithm}

Based on the discrete wavelet transform algorithm optimization, in this paper, we propose a method for further optimization of its threshold. For the one-dimensional signal $f(t)$, we first take discrete sample and collect discrete signal $f(n), n=0,1, \ldots, N-1$ from $N$ points. Its wavelet transform is:

$$
W T_{f}(j, k)=2^{-j / 2} \sum_{n=0}^{N-1} f(n) \psi\left(2^{-j} n-k\right)
$$

where $W T_{f}(j, k)$ is wavelet coefficient. It is complicated if use Eq. (27) directly for calculation in the real situation. Also, $\psi(t)$ usually has no expression. Therefore, based on the double dimension equation, the wavelet transform method of recursive implementation can be expressed as:

$$
\begin{aligned}
& S F(j+1, k)=S F(j, k) * h(j, k) \\
& W T_{f}(j+1, k)=S F(j, k)^{*} g(j, k)
\end{aligned}
$$

where $h$ and $g$ is low-pass and high-pass filter of scaling function $\varphi(x)$ and wavelet function $\psi(t)$, respectively. $S F(0, k)$ is original signal of $f(k) . S F(j, k)$ is scale factor. $W T_{f}(j, k)$ is wavelet coefficient. The wavelet transform reconstruction formula can be expressed as:

$$
S F(j-1, k)=S F(j, k)^{*} \tilde{h}(j, k) W T_{f}(j, k)^{*} \tilde{g}(j, k)
$$

For convenience, wavelet coefficient $W T_{f}(j, k)$ has been denoted as $w_{j, k}$. From the linear properties of wavelet transform, there are two parts existence at wavelet coefficient $w_{j, k}$ after discrete wavelet transform of $f(k)=s(k)+n(k)$. One part is the $s(k)$ 's wavelet coefficient $S s(j, k)$ denoted as $u_{j, k}$. Another part is $n(k)$ 's wavelet coefficient $\operatorname{Sn}(j, k)$ denoted as $v_{j, k}$.

The wavelet threshold value can be divided into soft threshold and hard threshold. In hard threshold, because the $\hat{w}_{j, k}$ is discontinuous at $\lambda$, the reconstructed signal may produce some oscillation. The induced from $\hat{w}_{j, k}$ soft threshold has overall good continuity, but when $\left|w_{j, k}\right| \geq \lambda$, here is a constant difference between $\hat{w}_{j, k}$ and $w_{j, k}$, which could directly affect the reconstructed signal and real signal approximation degree. Therefore, this article proposes a strategy for threshold compromise optimization. Set the wavelet coefficient as:

$$
\hat{w}_{j, k}\left\{\begin{array}{l}
\operatorname{sign}\left(w_{j, k}\right)\left(\left|w_{j, k}\right|-\alpha \lambda\right),\left|w_{j, k}\right| \geq \lambda \\
0, \quad\left|w_{j, k}\right|<\lambda
\end{array},(0 \leq \alpha \leq 1)\right.
$$

The absolute value of $\hat{w}_{j, k}$ induced from soft threshold has less than $w_{j, k}$ for $\lambda$, so reduction of the deviation is necessary. However, if reduce this value to 0 (as hard threshold) may also face some problems. Because the $w_{j, k}$ is constructed by $u_{j, k}$ and $v_{j, k},\left|w_{j, k}\right|>\left|u_{j, k}\right|$ may happen by the influence of $v_{j, k}$. However, our aim is to obtain the minimum value of $\left\|\hat{w}_{j, k}-u_{j, k}\right\|$. Therefore, put $\left|\hat{w}_{j, k}\right|$ between the $\left|w_{j, k}\right|-\lambda$ and $\left|w_{j, k}\right|$ may result a wavelet coefficient $\hat{w}_{j, k}$ more close to the $u_{j, k}$. Based on this idea, we add $\alpha$ factor in threshold estimator. A better performance can be obtained by appropriate adjustment of $\alpha$ between 0 and 1 . In this case, we use $\alpha=0.5$.

\subsection{Signal Denoising Based on Improved Wavelet Transform}

The denoising processing of weak signal chaotic detection system is based on the improved wavelet transform algorithm. Set the sampling signal as $s(n)=a \cos \left(\omega n / f_{s}+\varphi\right)+z(n)$, where $f_{s}$ is the sample frequency. The detail of denoising processing can be explained as follows:

Get signal $P s(n)$ after wavelet pretreatment of the sampled signal $s(n)$. Then $P s(n)$ has been $N$ finite discrete decomposition by improved wavelet transform algorithm to obtain the high frequency coefficient matrix:

$$
\left(\begin{array}{l}
C^{N} \\
D^{N} \\
D^{N-1} \\
M \\
D^{1}
\end{array}\right)=\left(\begin{array}{l}
L^{N} L^{N-1} L L^{1} \\
H^{N} L^{N-1} L L^{1} \\
H^{N-1} L^{N-2} L L^{1} \\
M \\
H^{1}
\end{array}\right) \operatorname{Ps}(n)
$$

where $P$ is improved wavelet pretreatment matrix. $D^{j}$ is $j$ 's high frequency coefficient matrix generated by wavelet transform algorithm. $H^{j}$ and $L^{j}$ is $j$ 's high frequency transformation matrix and low frequency transformation matrix.

Set $D^{j}(k, i)$ as the $i$ element in the row of $k$ in matrix $D^{j}$, representing coefficient $k$ in the high frequency subband of $i$ in the wavelet transform $j$. Considering the keep of signal energy, denoising threshold compromise strategy has been applied for $D^{j}(i, k)$ to get the treated wavelet transform coefficient $\hat{D}^{j}(i, k)$ :

$\hat{D}^{j}(i, k)=\left\{\begin{array}{l}D^{j}(i, k),\left|D^{j}(i, k)\right| \geq \lambda_{i}^{j} \\ 0,\left|D^{j}(i, k)\right|<\lambda_{i}^{j}\end{array}\right.$

where the value of $\lambda_{i}^{j}$ is decided by threshold compromise strategy. 
Denoising signal $\hat{s}(n)$ can be obtained by wavelet reconstruction of threshold processed wavelet coefficient $\hat{D}^{j}$ with $N$ :

$\hat{s}(n)=Q\left(\begin{array}{l}L^{N} L^{N-1} L L^{1} \\ H^{N} L^{N-1} L L^{1} \\ H^{N-1} L^{N-2} L L^{1} \\ M \\ H^{1}\end{array}\right)\left(\begin{array}{l}C^{N} \\ \hat{D}^{N} \\ \hat{D}^{N-1} \\ M \\ \hat{D}^{1}\end{array}\right)$

In the equation, $Q$ is post-processed wavelet transform matrix.

The denoising signal $\hat{s}(n)$ after autocorrelation arithmetic:

$$
R_{\hat{s} \hat{s}}(k)=\sum_{n} \hat{s}(n) \hat{s}(n-k)
$$

In order to facilitate subsequent iteration process, denote $R_{\hat{s} \hat{s}}(k)=R_{\hat{s} \hat{s}}(n)$.

Set Duffing vibrator parameters. Adjust the frequency of internal signals as $\omega$, target detect frequency as $\omega_{0}$. Set the cycle of the oscillator power amplitude threshold value for the system as $r_{1}$, letting the maximum Lyapunov index $\lambda$ meets $|\lambda| \leq 0.09$. The following Duffing oscillator is constructed:

$$
\left\{\begin{array}{l}
x=\omega_{0} y \\
y=\omega_{0}\left(x^{3}-x^{5}-k y+r_{1} \cos \left(\omega_{0} t\right)\right)
\end{array}\right.
$$

Put the signal $R_{\hat{s} \hat{s}}(n)$ into discrete Duffing oscillator:

$$
\left\{\begin{array}{l}
x\left(n / f_{s}\right)=\omega_{0} y\left(n / f_{s}\right) \\
y\left(n / f_{s}\right)=\omega_{0}\left(x^{3}\left(n / f_{s}\right)-x^{5}\left(n / f_{s}\right)-k y\left(n / f_{s}\right)\right. \\
\left.\quad+r_{1} \cos \left(\omega_{0}\left(n / f_{s}\right)+R_{\hat{s} s}(n)\right)\right)
\end{array}\right.
$$

where $f_{s}$ is sampling frequency of $s(n)$.

\section{Algorithm Performance Simulation}

In order to verify the effectiveness of the improved algorithm proposed in this paper, simulation experiments are conducted in the PC of memory with $2 \mathrm{~GB}$, and CPU as Pentium (R) Dual - Core T4200 1.2 GHZ. The results of denoising processing using traditional wavelet transform algorithm and improved wavelet transform algorithm are presented in Table 1 and Fig. 1.

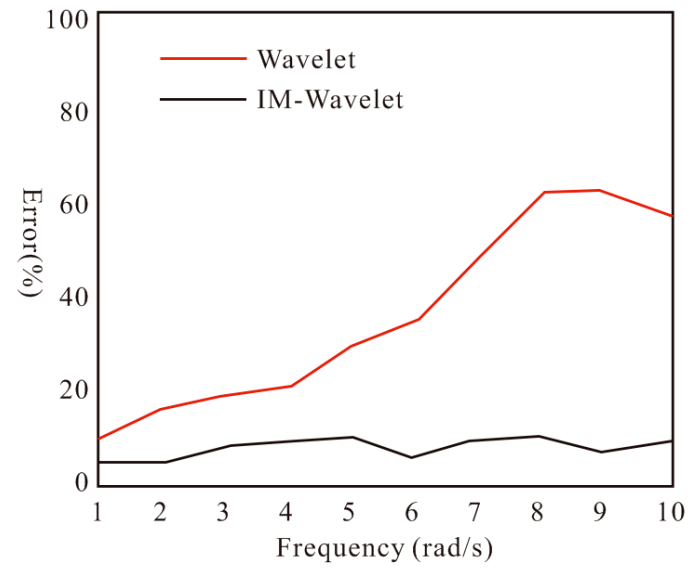

Fig. 1. Comparison of two algorithms de-noising error.

\begin{tabular}{cccccc}
\hline \multirow{2}{*}{$\begin{array}{c}\text { Zrequency } \\
\text { (rad/s) }\end{array}$} & \multicolumn{2}{c}{ SNR/dB } & Relative & \multicolumn{2}{c}{ Time/s } \\
Wavelet & [- Wavelet & error & Wavelet & [- Wavelet \\
\hline 1 & 6.97 & 6.17 & $12.52 \%$ & 1.24 & 0.55 \\
\hline 2 & 7.65 & 6.35 & $12.63 \%$ & 1.94 & 0.93 \\
\hline 3 & 8.25 & 6.77 & $14.84 \%$ & 3.25 & 1.61 \\
\hline 4 & 8.34 & 6.94 & $16.15 \%$ & 4.05 & 1.90 \\
\hline 5 & 9.43 & 7.03 & $17.74 \%$ & 6.18 & 2.47 \\
\hline 6 & 9.78 & 7.25 & $21.35 \%$ & 9.41 & 3.04 \\
\hline 7 & 10.23 & 7.63 & $24.63 \%$ & 12.83 & 3.73 \\
\hline 8 & 10.52 & 7.90 & $27.35 \%$ & 15.04 & 5.17 \\
\hline 9 & 11.13 & 8.14 & $29.47 \%$ & 19.01 & 6.32 \\
\hline 10 & 11.79 & 8.98 & $31.67 \%$ & 24.86 & 7.94 \\
\hline
\end{tabular}

Tab. 1. Wavelet transform algorithm and improved contrast wavelet transform algorithm.

Then the improved wavelet transform algorithm is used in the weak signal detection chaotic system. Fig. 2 shows the weak signal without noise. Fig. 3 shows the weak signal with noise. Fig. 4 shows the denoising results of weak signal detection chaotic system based on the wavelet transform. Fig. 5 shows the denoising results of weak signal detection chaotic system based on the improved wavelet transform. The statistical results are shown in Table 2.

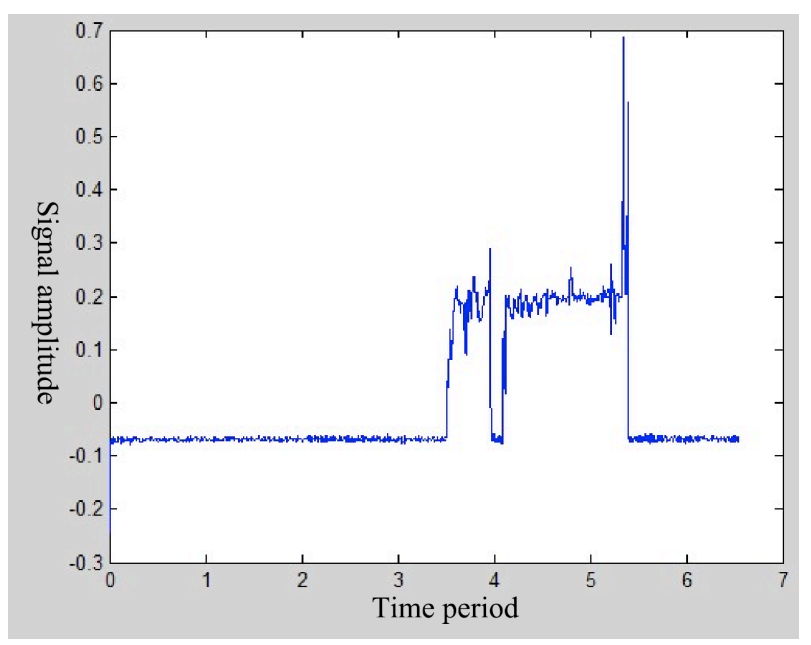

Fig. 2. The weak signal-to-noise-free. 


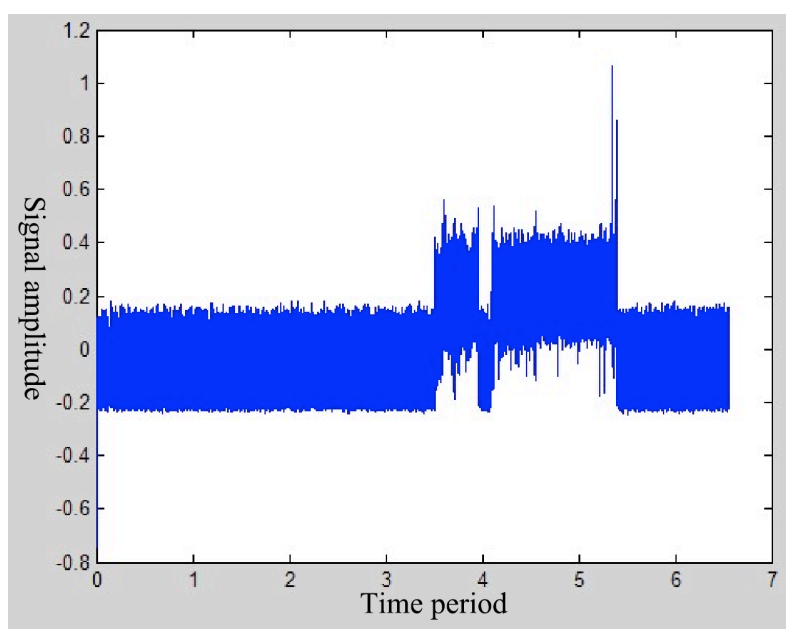

Fig. 3. Containing a weak signal-to-noise.

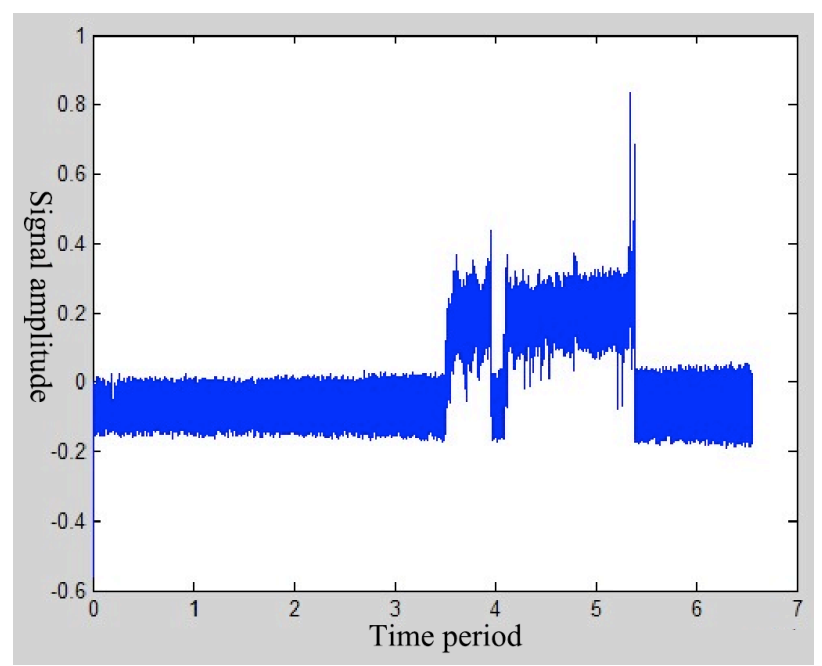

Fig. 4. Denoising result of chaotic detection system based on wavelet transform.

\begin{tabular}{cccc}
\hline \multirow{2}{*}{$\begin{array}{c}\text { Frequency } \\
(\mathrm{rad} / \mathrm{s})\end{array}$} & \multicolumn{2}{c}{ SNR/dB } & \multirow{2}{*}{ Relative error } \\
\cline { 2 - 3 } & Wavelet & IM- Wavelet & \\
\hline 1 & 3.25 & 2.11 & $31.4 \%$ \\
2 & 4.64 & 2.36 & $30.4 \%$ \\
3 & 4.91 & 2.53 & $35.2 \%$ \\
4 & 5.45 & 2.70 & $38.2 \%$ \\
5 & 5.79 & 3.12 & $38.3 \%$ \\
6 & 6.48 & 3.38 & $35.9 \%$ \\
7 & 7.39 & 4.69 & $36.1 \%$ \\
8 & 8.90 & 5.71 & $30.5 \%$ \\
9 & 10.26 & 6.91 & $30.8 \%$ \\
10 & 11.49 & 8.26 & $32.6 \%$ \\
\hline
\end{tabular}

Tab. 2. Error analysis of weak signal denoising.

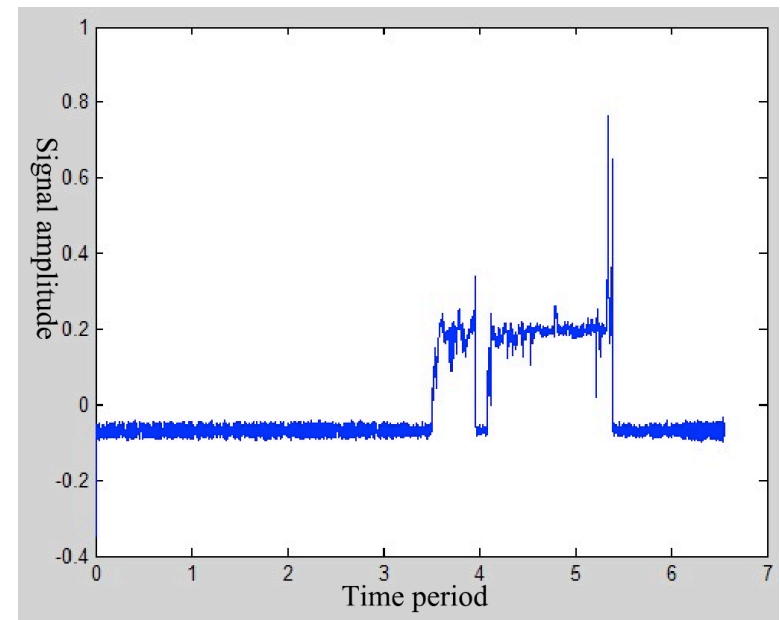

Fig. 5. Denoising result of chaotic detection system based on improved wavelet transform.

It can be seen from the simulation results that the discrete wavelet transform algorithm of optimization and compromise threshold strategy presented in this paper show better denoising effect than the original algorithm. It improves the denoising effect when applying to the weak signal chaotic detection system.

\section{Conclusion}

Weak signal detection (WSD) is a new technique rising in recent years, which overcomes the problem of high input SNR threshold in traditional linear signal detection technology. This paper proposes a weak signal chaotic detection system based on wavelet transform algorithm, optimizing the wavelet transform algorithm with discrete optimization and threshold compromise strategy. It can be seen from the simulation results comparing with traditional wavelet transform that the improved strategy of this paper increases the detection accuracy of weak signal with noise and shows the better denoising effect than the original algorithm.

\section{References}

1. H. Xing, Dissipation type synchronous weak periodic signal detection and noise impact analysis, Journal of Jilin University, vol. 4 , pp. 1182-1190 (2014).

2. A. Arneodo, P. Coullet, and C. Tresser, Possible new strange attractors with spiral structure, Communications in Mathematical Physics, vol. 79, pp. 573-579 (1981).

3. L. Pang, Research and development of the data collector for time domain aeronautical electromagnetic weak signal, Process Automation Instrumentation, vol. 35, pp. 83-86 (2014).
4. W. Liu, A general amplifier design for low frequency weak signal acquisition, Journal of UEST of China, vol. 2, pp. 222225 (2014).

5. C. Zhang, Analyses based on the technology of high-speed weak photoelectric detection related research, Science Technology and Engineering, vol. 8, pp. 182-185 (2014).

6. Klein, Research on weak signal detection in time domain based on phase locked loop and Duffing oscillator, Science Technology and Engineering, vol. 6, pp. 13-9 (2014). 
7. Weinred, Research on chaotic characteristics of a deformable Rossler systems and its usage for weak signal detection, Computer Measurement \& Control, vol. 22(2), pp. 339-342 (2014).

8. Fourier, Weak signal detection system for marine environment based on LabVIEW, Instrument Technique and Sensor, vol. 11, pp. 35-37 (2013).

9. Wang, Weak sine signal detection based on improved double differential oscillators, Journal of Henan Normal University (Natural Science), vol. 6, pp. 57-60 (2013).

10. Y. Li, Chaos weak signal detection based on GPU critical threshold is determined, Application Research of Computers, vol. 31(4), pp. 1051-1054 (2014).

11. P. Lu, Chaos algorithm and subspace algorithm applied in weak signal detection, Science Technology and Engineering, vol. 1, pp. 235-238 (2014)
12. W. Chen, Research on weak signal detecting in distributed FBG sensing system, Journal of Xian University of Technology, vol. 29(4), pp. 428-433 (2014).

13. C. Li, Based on Morlet filter and the particle swarm algorithm of weak signal detection, Computer Measurement \& Control, vol. 21(10), pp. 2655-2657 (2013).

14. G. Wang, Weak signal intelligent detection system based on stochastic resonance and artificial fish swarm algorithm, Chinese Journal of Scientific Instrument, vol. 34(11), pp. 2464-2470 (2013).

15. M. Chance Glenn and S Hayes, The design of weak signal detection system in Strong magnetic field, Journal of Transduction Technology, vol. 26(6), pp.865-870 (2013). 